\title{
Anomalías morfológicas en los dientes del cibario de Lutzomyia evansi (Diptera: Psychodidae) en el estado Trujillo, Venezuela
}

\author{
Yolanda Méndez-de Daboín', Milagros Oviedo-Araújo, Adalberto González-Pérez', \\ Jorge Suárez-Hernández ${ }^{1}$, Claudia M. Sandoval², Dalmiro Cazorla ${ }^{3}$ \\ 1 Laboratorio de Biología de Lutzomyia e Insectario "Pablo Anduze", Instituto Experimental "José Witremundo \\ Torrealba", Universidad de Los Andes, Trujillo, República Bolivariana de Venezuela \\ 2 Laboratorio de Ecología Sensorial, Centro Multidisciplinario de Ciencias, Instituto Venezolano de Investigaciones \\ Científicas (IVIC), Mérida, República Bolivariana de Venezuela \\ ${ }^{3}$ Laboratorio de Entomología, Parasitología y Medicina Tropical (LEPAMET), Centro de Investigaciones \\ Biomédicas, Universidad Nacional Experimental "Francisco de Miranda", Falcón, República Bolivariana de
} Venezuela

Introducción. Lutzomyia evansi es un reconocido vector de Leishmania infantum en Colombia y Venezuela.

Objetivo. Describir e ilustrar las anomalías morfológicas presentes en el cibario de hembras de Lu. evansi capturadas en un foco rural de leishmaniasis visceral en Trujillo, Venezuela.

Materiales y métodos. Para la captura de los flebótomos se utilizaron tres diferentes métodos (trampa Shannon, trampas de luz del tipo CDC y capturas en reposo). En la identificación taxonómica se siguió la clave de Young \& Duncan (1994) y los diseños biológicos se hicieron utilizando un microscopio óptico con cámara clara.

Resultados. En 3.477 especímenes de Lu. evansi se detectaron cuatro $(0,12 \%)$ hembras con diferentes anomalías en el cibario.

Conclusión. Algunos especímenes de Lu. evansi pueden presentar anomalías morfológicas relacionadas con aumento del número de los dientes en el cibario y con su disposición. La detección de estas anomalías en poblaciones naturales de Lu. evansi puede evitar dificultades y confusiones en el momento de la identificación taxonómica de los especímenes teratomorfos, reduciendo así el riesgo de incurrir en errores taxonómicos.

Palabras clave: Psychodidae, teratología, leishmaniasis visceral, clasificación, Venezuela.

doi: http://dx.doi.org/10.7705/biomedica.v35i2.2552

Morphological abnormalities in the cibarium of Lutzomyia evansi (Diptera: Psychodidae, Phlebotominae) caught in Trujillo, Venezuela

Introduction: Lutzomyia evansi is a recognized vector of Leishmania infantum in Colombia and Venezuela.

Objective: To describe and illustrate the morphological abnormalities in Lu. evansi females captured in a rural focus of visceral leishmaniasis in Trujillo, Venezuela.

Materials and methods: Phlebotomine sand flies were collected using CDC light traps, Shannon traps and aspiration in resting places. The identification was performed according to Young \& Duncan (1994) and drawings were made using a microscope with camara lucida.

Results: Abnormalities in the cibarium of $L u$. evansi were detected in $4(0.12 \%)$ females of the 3,477 adults that were studied.

Conclusion: Lutzomyia evansi can have uncommon morphological variants associated with an increase in the number of teeth in the cibarium and their arrangement, which may lead to errors in the taxonomic identification of anomalous specimens. The study of such deformities can serve to avoid taxonomic identification errors.

Key words: Teratology, phlebotomine, visceral leishmaniasis, taxonomy.

doi: http://dx.doi.org/10.7705/biomedica.v35i2.2552

\section{Contribución de los autores:}

Yolanda Méndez, Milagros Oviedo, Dalmiro Cazorla y Claudia M. Sandoval: taxonomía de flebótomos y redacción del manuscrito Adalberto González y Jorge Suárez: trabajo de campo

Dalmiro Cazorla: diseño biológico de los cibarios 
La incidencia de la leishmaniasis visceral en el mundo se estima anualmente en 400.000 nuevos casos, de los cuales el $90 \%$ se reporta en Bangladesh, Brasil, Etiopía, India y Sudán (1). Es una infección oportunista emergente asociada con el HIV y su tasa de fatalidad puede oscilar entre 10 y $20 \%$ (2).

En el continente americano, los principales vectores del protozoario flagelado Leishmania (L.) infantum, agente etiológico de la leishmaniasis visceral, son Lutzomyia longipalpis y Lutzomyia evansi (3). En Venezuela, esta enfermedad es endémica y está focalizada, con una incidencia media anual de 0,2 casos por 100.000 habitantes $(4,5)$. En el estado Trujillo, ubicado en la región andina, se estima una media anual de 0,5 casos por 100.000 habitantes. Además de Lu. longipalpis, la presencia de $L u$. evansi es común en las áreas rurales con focos activos de transmisión de la enfermedad (6).

Por otra parte, en diversos estudios taxonómicos de los Phlebotominae encontrados en los distintos focos de transmisión, se han encontrado anomalías morfológicas en especies de los géneros Lutzomyia (Nuevo Mundo) y Phlebotomus (Viejo Mundo). Estas anomalías, incluyendo las merísticas, pueden hallarse en los genitales masculinos o femeninos, en las patas, las alas, las antenas y en los palpos, así como en las estructuras internas de la cabeza (7-11). Varios autores coinciden en señalar que la presencia de anomalías puede acarrear o inducir a error en la correcta identificación de las especies (12-14). Por tal motivo, este trabajo se centró en la detección y la descripción de anomalías morfológicas en el cibario de los flebótomos, para lo cual se aprovechó el material entomológico obtenido en estudios previos llevados a cabo por el equipo de trabajo en un área de transmisión de leishmaniasis visceral en Venezuela.

\section{Materiales y métodos}

El área de estudio se ubica en la localidad de Montañas de Peraza (1044'50" N; 33²5’00” O), municipio Pampanito, estado Trujillo, en la región

\footnotetext{
Correspondencia:

Milagros Oviedo, Laboratorio de Biología de Lutzomyia e Insectario "Pablo Anduze", Instituto Experimental "José Witremundo Torrealba", Núcleo Universitario Rafael Rangel, Sede Carmona, Av. Angarita, Trujillo, República Bolivariana de Venezuela.

Teléfono: (0272) 2362177

lulongipalpis@yahoo.es

Recibido: 03/10/14; aceptado: 12/03/15
}

andina venezolana. Es un área rural con transmisión activa de leishmaniasis visceral, caracterizada como una región bioclimática de bosque seco tropical, localizada a $403 \mathrm{msnm}$, con temperatura media anual de $26,5^{\circ} \mathrm{C}$, precipitación media anual de $1.386 \mathrm{~mm}$ y humedad relativa de $60 \%$ (15).

Durante el 2005 y el 2006, se hicieron 24 muestreos entomológicos con una frecuencia mensual, y en el 2008 se llevaron a cabo muestreos bimensuales en un lapso de seis meses, en esta localidad. Se incluyeron los flebótomos capturados en 34 muestreos entomológicos, cuyo propósito principal fue la captura de especímenes para estudios de competencia vectorial y aspectos ecológicos de los flebótomos implicados en la transmisión de la leishmaniasis visceral (15).

Los métodos de captura utilizados en cada muestreo, fueron los siguientes: una trampa Shannon colocada entre las 19:00 y 21:00 horas en el peridomicilio, dos trampas de luz del tipo CDC colocadas en el domicilio y en el peridomicilio entre las 19:00 y las 06:00 horas, y capturas en reposo por aspiración directa en abrigos naturales entre las 15:00 y las 17:00.

Los flebótomos capturados se transportaron al laboratorio en jaulas de organdí dispuestas en recipientes de poliestireno. Posteriormente, los insectos se trataron con una solución de Tritón X-100 (0,4\%), y se embebieron en una solución de Nesbitt durante 24 horas; para los montajes se utilizó el medio de Berlese (16).

La identificación y la clasificación taxonómica de los flebótomos se hizo según los criterios de la clave dicotómica de Young y Duncan (1994) (17). Para el diseño y la descripción de estas anomalías, se efectuaron dibujos en 40X en un microscopio óptico marca Leica con cámara clara. Los montajes reposan en la colección de flebótomos del Laboratorio de Biología de Lutzomyia e Insectario Pablo Anduze.

\section{Resultados}

Se capturaron 4.231 flebótomos, distribuidos en ocho especies de Lutzomyia (cuadro 1), de los cuales el 82,2 \% (3.477/4.231) pertenecía a la especie Lu. evansi, seguida de Lu. ovallesi, con 12,07\% (511/4.231). El carácter merístico reconocido como normal en el cibario de las hembras de Lu. evansi, es la presencia de cuatro dientes horizontales dispuestos en hilera, todos con un tamaño similar (17). 
Cuadro 1. Especies de Lutzomyia identificadas en Montañas de Peraza, Trujillo, Venezuela, en capturas con diferentes métodos durante 2005, 2006 y 2008

\begin{tabular}{lrrrrrrrr}
\hline Especie & SH & \% & CDC & $\%$ & R & $\%$ & Total & $\%$ \\
\hline Lu. evansi & 2.175 & 95,31 & 855 & 62,09 & 447 & 78,15 & 3.477 & 82,2 \\
Lu. ovallesi & 53 & 2,32 & 454 & 32,97 & 4 & 0,70 & 511 & 12,07 \\
Lu. panamensis & 20 & 0,88 & 37 & 2,68 & 1 & 0,17 & 58 & 1,37 \\
Lu. walkeri & 17 & 0,74 & 9 & 0,65 & 0 & 0,00 & 26 & 0,61 \\
Lu. gomezi & 13 & 0,57 & 15 & 1,08 & 5 & 0,87 & 33 & 0,77 \\
Lu. scorzai & 2 & 0,09 & 0 & 0 & 0 & 0,00 & 2 & 0,04 \\
Lu. venezuelensis & 1 & 0,04 & 0 & 0 & 0 & 0,00 & 1 & 0,02 \\
Lu. trinidadensis & 1 & 0,04 & 1 & 0,07 & 41 & 7,17 & 43 & 1,06 \\
Lu. hernandezi & 0 & 0,00 & 3 & 0,21 & 0 & 0,00 & 3 & 0,07 \\
Lu. shannoni & 0 & 0,00 & 2 & 0,14 & 0 & 0,00 & 2 & 0,04 \\
Lu. dubitans & 0 & 0,00 & 1 & 0,07 & 0 & 0,00 & 1 & 0,02 \\
Lu. atroclavata & 0 & 0,00 & 0 & 0 & 30 & 5,24 & 30 & 0,7 \\
Lu. cayennensis & 0 & 0,00 & 0 & 0 & 25 & 4,37 & 25 & 0,59 \\
Lu. micropyga & 0 & 0,00 & 0 & 0 & 9 & 1,57 & 9 & 0,21 \\
Lu. pilosa & 0 & 0,00 & 0 & 0 & 9 & 1,57 & 9 & 0,21 \\
Lu. rangeliana & 0 & 0,00 & 0 & 0 & 1 & 0,17 & 1 \\
Lu. lichyi & 0 & 0,00 & 0 & 0 & 0 & 0,00 & 0,02 \\
Totales & $\mathbf{2 8 2}$ & $\mathbf{1 0 0}$ & $\mathbf{1 . 3 7 7}$ & $\mathbf{1 0 0}$ & $\mathbf{5 7 2}$ & $\mathbf{1 0 0}$ & $\mathbf{4 . 2 3 1}$ & $\mathbf{1 0 0}$ \\
\hline
\end{tabular}

SH: trampa Shannon; CDC: trampas de luz del tipo CDC; R: capturas en reposo por aspiración directa.

En este trabajo se reportan cuatro diferentes anomalías morfológicas, todas encontradas en el cibario de las hembras de Lu. evansi que, además de las variantes merísticas, incluían algunos cambios en la posición y el tamaño de los dientes (figura 1). El porcentaje de anomalías en el cibario registrado para la especie $L u$. evansi en el área de estudio, correspondió a 0,12 \% (4:3.477).

La primera anomalía corresponde a la presencia de un diente horizontal adicional, para un total de cinco dientes horizontales de similar tamaño (figura 1A). La segunda anomalía corresponde a un total de siete dientes, de los cuales cinco son de similar tamaño, en tanto que el sexto, ubicado en el lateral derecho, y el séptimo, ubicado en el lateral izquierdo y desfasado de la línea horizontal, aparecen de menor tamaño (figura 1B). La tercera anomalía corresponde a la presencia de seis dientes, cuatro dispuestos en la línea horizontal y dos de menor tamaño, ubicados debajo y en el centro (figura 1C). La cuarta anomalía corresponde a cinco dientes horizontales, cuatro dispuestos en la línea horizontal y de tamaño similar, y uno pequeño ubicado debajo y en el centro (figura 1D).

\section{Discusión}

Las anomalías, especialmente en los caracteres de tipo merístico del cibario, han sido descritas previamente en hembras de las especies $L u$. youngi, Lu. lichy, Lu. gomezi, Lu. trinidadensis, Lu. atroclavata, Lu. ovallesi y Lu. intermedia $(7,12)$. En

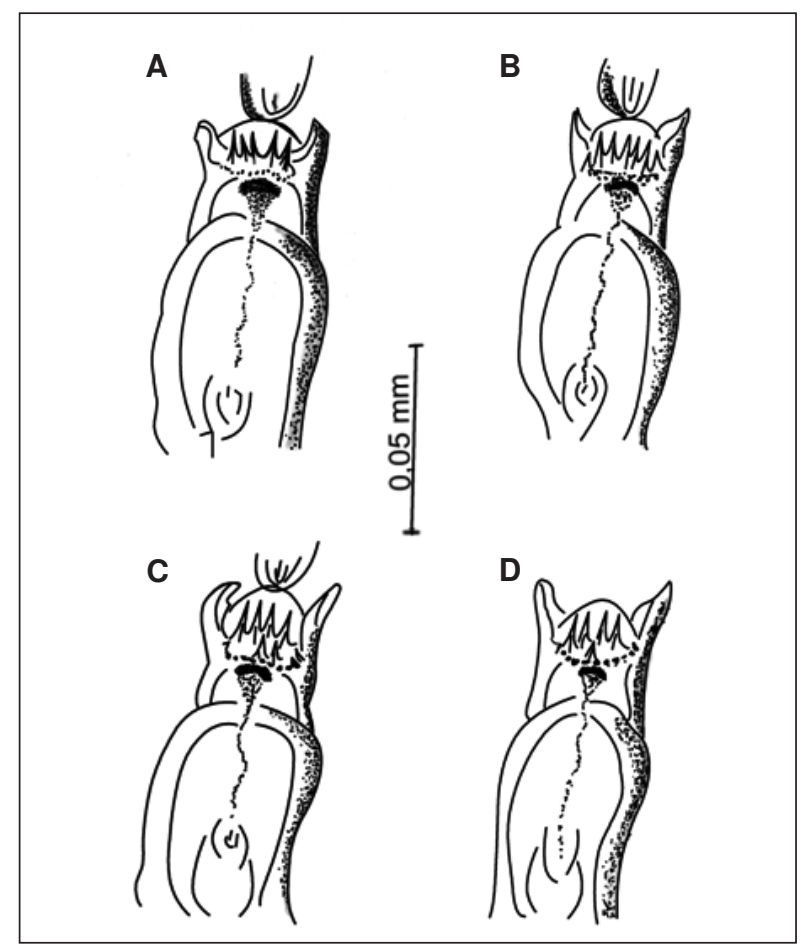

Figura 1. Cibarios de Lutzomyia. evansi con anomalías detectadas en el número, tamaño y disposición de los dientes horizontales. A) Cinco dientes horizontales de tamaño similar. B) Siete dientes, cinco de igual tamaño y dos adicionales de menor tamaño, ubicados en los laterales, uno de ellos desfasado de la línea horizontal. C) Seis dientes, cuatro dispuestos en la línea horizontal de similar tamaño y dos pequeños ubicados debajo y en el centro. D) Cinco dientes, cuatro de similar tamaño dispuestos en la línea horizontal, y uno pequeño ubicado debajo y en el centro. 
Lu. evansi se conoce la presencia de alteraciones morfológicas en especímenes de una población de la costa caribe colombiana, pero relacionadas con la aparición de una tercera espermateca (18).

El reporte de cuatro diferentes anomalías en el número y la disposición de los dientes del cibario en hembras de Lu. evansi tiene importancia taxonómica, pues se trata de cambios en un carácter diagnóstico útil en la separación de las especies del grupo Verrucarum (17), el cual aparece en una estructura impar, lo que implica que no puede resolverse fácilmente con el examen de la estructura correspondiente, como generalmente sucede en las mutaciones presentes en los segmentos de antenas, palpos y de los genitales masculinos $(9,10,19)$, y obstaculiza hasta cierto punto el proceso de identificación. Sin embargo, es necesario mencionar que los dientes del cibario no son un carácter determinante en el nivel específico (17), y la detallada inspección de las espermatecas y otros caracteres, como la coloración de ciertas regiones del tórax y las relaciones métricas de algunos apéndices de la cabeza, permitieron la correcta identificación taxonómica de los especímenes teratomorfos de Lu. evansi en el área de estudio.

En conclusión, consideramos que, aun cuando la frecuencia de las anomalías en el cibario de $L u$. evansi es baja, su reporte es pertinente, pues el conocimiento de su presencia en poblaciones naturales puede evitar dificultades y eventuales confusiones en el proceso de la identificación taxonómica de algunos especímenes $(10,19)$, además de contribuir a reducir el riesgo de aparición de nuevas sinonimias (9), ya que en otros grupos de insectos se ha demostrado que las anomalías pueden ser fuente de errores taxonómicos (20).

\section{Conflicto de intereses}

Ninguno de los autores declara conflictos de interés para la publicación del presente manuscrito.

\section{Financiación}

El trabajo fue financiado por FONACIT, Proyecto S-01-20000 51, y por la Misión Ciencia del Ministerio del Poder Popular para Ciencia, Tecnología e Innovación.

\section{Referencias}

1. World Health Organization. Control of the leishmaniasis: Report of a meeting of the WHO Expert Committee on the Control of Leishmaniases, Geneva: WHO; 2010. p. 186.
2. Ready P. Epidemiology of visceral leishmaniasis. Clin Epidemiol. 2014;6:147-54. http://dx.doi.org/10.2147/CLEP. S44267

3. Montoya-Lerma J, Cadena H, Oviedo M, Ready PD, Barazarte R, Travi BL, et al. Comparative vectorial efficiency of Lutzomyia evansi and Lu. longipalpis for transmitting Leishmania chagasi. Acta Trop. 2003;85:19-29. http://dx.doi.org/10.1016/S0001-706X(02)00189-4

4. Zerpa O, Ulrich M, Borges R, Rodríguez V, Centeno M, Negrón E, et al. Epidemiological aspects of human and canine visceral leishmaniasis in Venezuela. Rev Panam Salud Pública. 2003;13:239-45. http://dx.doi.org/10.1590/ S1020-49892003000300006

5. Feliciangeli MD, Delgado $O$, Suárez $B$, Chiurillo MA. The burden of the Leishmania chagasi/infantum infection in a closed rural focus of visceral leishmaniasis in Lara state, west-central Venezuela. Trop Med Int Health. 2005;10:444-49. http://dx.doi.org/10.1111/j.13653156.2005.01408.x

6. Vásquez LC, Vásquez LR, Oviedo M, Sandoval C, Méndez Y, Bastidas G, et al. Perfil clínico y epidemiológico de la leishmaniasis visceral americana en el estado Trujillo, Venezuela (1975-2007). Bol Dir Malariol San Amb. 2010;50:233-42.

7. Marcondes CB. Anomalies of Lutzomyia intermedia (Lutz \& Neiva, 1912) (Diptera, Psychodidae, Phlebotominae). Mem Inst Oswaldo Cruz. 1999;94:365-6. http://dx.doi. org/10.1590/S0074-02761999000300014

8. Ximenes MF, Castellón EG, Freitas RA. Morphological genitalic anomaly in specimens Lutzomyia sensu lato (Lutz \& Neiva, 1912) collected in the State of Rio Grande do Norte, Brazil. Entomotropica. 2002;17:183-4.

9. Andrade Filho JD, de Lima Carvalho GM, Saraiva L, Lima Falcão A. Bilateral anomaly in the style of Micropygomyia schreiberi (Martins, Falcão \& Silva) (Diptera, Psychodidae). Rev Bras Entomol. 2004;48:583-5. http://dx.doi.org/10.1590/ S0085-56262004000400024

10. Estrada L, Flórez F, Bejarano E. Cambios teratológicos en flebotomíneos (Diptera: Psychodidae) del subgénero Micropygomyia Barretto, 1962. Revista Colombiana de Ciencia Animal. 2009;1:216-21.

11. Srinivasan R, Jambulingam P. Morphological and anatomical variations among Phlebotomus (Phlebotomus) papatasi sensu lato (Diptera: Psychodidae). J Med Entomol. 2012;49:441-4.

12. Cazorla D, Añez E, Nieves E. Variaciones morfológicas en cuatro especies de flebotominos (Diptera: Psychodidae) del estado Mérida, Venezuela. Bol Dir Malariol San Amb. 1988;28:91-8.

13. Guernaoui S, Ramaoui K, Rahola N, Barnabe C, Sereno D, Boumezzough A. Malformations of the genitalia in male Phlebotomus papatasi (Scopoli) (Diptera: Psychodidae). J Vector Ecol. 2010;35:13-9. http://dx.doi.org/10.1111/j.19487134.2010.00022.x.

14. Kassem H, Abdel S, Shehata MG, el Sawaf BJ. Abnormalities in the sand fly Phlebotomus papatasi (Scopoli) (Diptera: Psychodidae) of Egypt. Egypt Public Health Assoc. 1988;63:209-13. 
15. Méndez Y. Diseño de un mapa de riesgo ecoepidemiológico de la leishmaniosis visceral americana en el estado Trujillo Venezuela (SIG-Lv) (tesis). Trujillo: Universidad de Los Andes; 2012

16. Maroli M, Feliciangeli MD, Arias J. Métodos de captura, conservación y montaje de los flebótomos (Diptera: Psychodidae). Washington, D.C.: OPS; 1997. p. 1-67.

17. Young DG, Duncan MA. Guide to the identification and geographic distribution of Lutzomyia sand flies in Mexico, the West Indies, Central and South America (Diptera: Psychodidae). Mem Am Entomol Inst. 1994;54:1-881.
18. Pérez-Doria A, Bejarano EE. Teratología en el vector de leishmaniosis visceral Lutzomyia evansi (Núñez-Tovar, 1924). Anal Biol. 2005;27:133-5.

19. Cutolo AA, Von Zuben CJ, Galati EA. Anomalia bilateral no gonóstilo de Sciopemyia sordellii (Shannon \& Del Ponte) (Diptera, Psychodidae). Rev Bras. Parasitol Vet. 2009;18:67-9. http://dx.doi.org/1010.4322/rbpv.01803012

20. Popovici OA, Mitroiu MD, Notton DG. New teratological cases in Platygastridae and Pteromalidae (Hymenoptera). Turk J Zool. 2014;38:491-9. http://dx.doi:10.3906/zoo1312-30 